\title{
LATE ELONGATED HYPOCOTYL regulates photoperiodic flowering via the circadian clock in Arabidopsis
}

\author{
Mi-Jeong Park ${ }^{1 \dagger}$, Young-Ju Kwon ${ }^{1 \dagger}$, Kyung-Eun Gil $^{1}$ and Chung-Mo Park ${ }^{1,2^{*}}$
}

\begin{abstract}
Background: Plants constantly monitor changes in photoperiod or day length to trigger the flowering cycle at the most appropriate time of the year. It is well established that photoperiodic flowering is intimately associated with the circadian clock in Arabidopsis. In support of this notion, many clock-defective mutants exhibit altered photoperiodic sensitivity in inducing flowering. LATE ELONGATED HYPOCOTYL (LHY) and its functional paralogue CIRCADIAN CLOCK ASSOCIATED 1 (CCA1) constitute the core of the circadian clock together with TIMING OF CAB EXPRSSION 1 (TOC1). While it is known that TOC1 contributes to the timing of flowering entirely by modulating the clock function, molecular mechanisms by which LHY and CCA1 regulate flowering time have not been explored.

Results: We investigated how LHY and CCA1 regulate photoperiodic flowering through molecular genetic and biochemical studies. It was found that LHY-defective mutants (Ihy-7 and Ihy-20) exhibit accelerated flowering under both long days (LDs) and short days (SDs). Consistent with the accelerated flowering phenotypes, gene expression analysis revealed that expression of the floral integrator FLOWERING LOCUS T (FT) is up-regulated in the Ihy mutants. In addition, the expression peaks of GIGANTEA (GI) and FLAVIN-BINDING, KELCH REPEAT, F-BOX PROTEIN 1 (FKF1) genes, which constitute the clock output pathway that is linked with photoperiodic flowering, were advanced by approximately $4 \mathrm{~h}$ in the mutants. Furthermore, the up-regulation of $F T$ disappeared when the endogenous circadian period is matched to the external light/dark cycles in the Ihy-7 mutant. Notably, whereas CCA1 binds strongly to FT gene promoter, LHY does not show such DNA-binding activity.

Conclusions: Our data indicate that the advanced expression phases of photoperiodic flowering genes are associated with the clock defects in the Ihy mutants and responsible for the reduced photoperiodic sensitivity of the mutant flowering, demonstrating that LHY regulates photoperiodic flowering via the circadian clock, similar to what has been shown with TOC1. It is notable that while LHY regulates photoperiodic flowering in a similar manner as with TOC1, the underlying molecular mechanism would be somewhat distinct from that exerted by CCA1 in Arabidopsis.
\end{abstract}

Keywords: Arabidopsis thaliana, Circadian clock, Flowering time, LHY, Photoperiod

\section{Background}

The appropriate timing of flowering is critical for reproductive success in plants. Since the transition from the vegetative to the reproductive phases is irreversible, plants precisely coordinate endogenous developmental signals and environmental cues, such as changes in photoperiod,

\footnotetext{
* Correspondence: cmpark@snu.ac.kr

${ }^{\dagger}$ Equal contributors

${ }^{1}$ Department of Chemistry, Seoul National University, Seoul 151-742, Korea

${ }^{2}$ Plant Genomics and Breeding Institute, Seoul National University, Seoul 151-742, Korea
}

(C) 2016 Park et al. Open Access This article is distributed under the terms of the Creative Commons Attribution 4.0 International License (http://creativecommons.org/licenses/by/4.0/), which permits unrestricted use, distribution, and reproduction in any medium, provided you give appropriate credit to the original author(s) and the source, provide a link to the Creative Commons license, and indicate if changes were made. The Creative Commons Public Domain Dedication waiver (http://creativecommons.org/publicdomain/zero/1.0/) applies to the data made available in this article, unless otherwise stated.

light quality and quantity, and temperature, to optimize the timing of flowering [1-3]. Both the endogenous and environmental signals are incorporated into flowering genetic pathways via the floral integrators, such as FLOWERING LOCUS T (FT) and SUPPRESSOR OF CONSTANS OVEREXPRESSION 1 (SOC1) [4, 5].

Photoperiod is a major environmental cue that profoundly affects floral induction [2, 3, 6]. Plants monitor photoperiodic changes to anticipate seasonal changes. CONSTANS (CO), which is a B-box zinc finger transcription factor [7], plays a central role in photoperiodic 
flowering by activating $F T$ expression $[8,9]$. Accordingly, $\mathrm{CO}$-defective mutants and CO-overexpressing plants exhibit photoperiod-insensitive flowering phenotypes $[10,11]$.

The photoperiod-sensitive $F T$ induction is mediated by the distinct accumulation peak of $\mathrm{CO}$ in late afternoon under long days (LDs), which is shaped by the coordinated actions of several ubiquitin/proteasome-dependent pathways [12]. A small group of E3 ubiquitin ligases and photoreceptors modulate the CO stability. The light signaling mediator CONSTITUTIVE PHOTOMORPHOGENIC 1 (COP1) degrades CO at night $[13,14]$. In the light phase of the day, two opposing regulations occur through the actions of HIGH EXPRESSION OF OSMOTICALLY RESPONSIVE GENES 1 (HOS1) and FLAVIN-BINDING, KELCH REPEAT, F-BOX 1 (FKF1) E3 ubiquitin ligases. HOS1 degrades $\mathrm{CO}$ in the morning, and FKF1 stabilizes CO in late afternoon $[15,16]$. The sequential actions of HOS1 and FKF1 contribute to the maintenance of $\mathrm{CO}$ accumulation at a basal level in the morning but at a high level in later afternoon, and thus Arabidopsis flowering is induced only under LDs [6]. Meanwhile, PHYTOCHROME B mediates $\mathrm{CO}$ degradation, but PHYTOCHROME $\mathrm{A}$ and CRYTOCHROME photoreceptors mediate CO stabilization [12]. It is notable that $\mathrm{CO}$ accumulation occurs at the specific time phase of the day under LDs, necessitating that photoperiodic flowering would be closely linked with the circadian clock $[2,3,17]$.

Many clock-defective Arabidopsis mutants exhibit alterations in the photoperiodic sensitivity of flowering time, supporting the intimate linkage between the clock and photoperiodic flowering [18-22]. In addition, the circadian clock regulates the rhythmic expression of photoperiodic flowering genes, such as CO, FKF1, GIGANTEA (GI), and CYCLING DOF FACTOR 1 (CDF1) [23-26]. The clock allows the high-level expression of $C O$ gene occurs in the light only under LDs [23]. The clock-controlled peak of FKF1 and GI expression in the LD afternoon renders FKF1-GI complex to be formed, which is crucial for $\mathrm{CO}$ accumulation [16]. On the other hand, the prevention of $C O$ and $F T$ expression by CDFs occurs in the morning through the clock function [26, 27]. It has been shown that the early flowering phenotypes of short-period plants, such as TIMING OF CAB EXPRESSION 1 (TOC1)-defective mutant (toc1) and CASEIN KINASE II BETA SUBUNIT 4 (CKB4)-overexpressing plants, are caused by the advanced expression peaks of photoperiodic flowering genes $[28,29]$.

While the altered flowering phenotypes of toc1 mutant and CKB4-overexpressing plants are entirely caused by clock defects, clock-independent control of photoperiodic flowering by clock components has also been proposed [30-32]. For instance, GI, which plays a role in regulating clock progression [33], activates $F T$ expression by directly binding to its gene promoter independent of $\mathrm{CO}$ [30]. It has recently been reported that SENSITIVITY TO RED
LIGHT REDUCED 1 (SRR1), which is required for normal oscillator function [34], regulates floral transition in a COindependent manner [31]. Similarly, DE-ETIOLATED 1 (DET1), which is a transcriptional corepressor important for clock progression, acts as a floral repressor [32]. It is notable that GI, SRR1, and DET1 regulate flowering time independent of their roles in the clock function. In addition, they do not require $\mathrm{CO}$, the central promoter of photoperiodic flowering.

LATE ELONGATED HYPOCOTYL (LHY) and its functional paralogue CIRCADIAN CLOCK ASSOCIATED 1 (CCA1) constitute the central oscillator in Arabidopsis $[20,35,36]$. Arabidopsis mutants that are defective in LHY and CCA1 exhibit early flowering even under noninductive conditions $[20,22,37]$. It has been shown that CCA1 regulates flowering time by binding to the SOC1 gene promoter [38]. However, it has not been explored at the molecular level how LHY regulates flowering time.

In this work, with an aim of clarifying the molecular mechanism by which LHY regulates flowering time, we performed molecular genetic and biochemical studies on LHY-defective mutants (lhy-7 and lhy-20). Notably, the expression peaks of photoperiodic flowering genes were shifted earlier in the lhy mutants. We found that the advanced expression phases of photoperiodic flowering genes are associated with the clock defects in the mutants and underlie the reduced photoperiodic sensitivity of the mutant flowering. By matching the external light/ dark cycles to the endogenous circadian period, the early flowering phenotype of the mutants was rescued. Interestingly, CCA1 binds strongly to the $F T$ gene promoter, but LHY does not exhibit such DNA-binding activity. Our data indicate that while LHY regulates the timing of flowering entirely via the circadian clock like TOC1, CCA1 would be functionally distinct from TOC1 and LHY in regulating photoperiodic flowering.

\section{Results \\ Loss-of-function Ihy mutants exhibit early flowering under both LDs and SDs}

To investigate the functional roles of LHY in photoperiodic flowering, we examined the flowering phenotypes of LHY-defective mutants. We also aimed to clarify the molecular mechanisms by which LHY regulates flowering time: whether LHY affects flowering time entirely by modulating the circadian rhythms like TOC1 [28] or regulating the expression of specific flowering genes like GI [30] or both. In additional to the previously reported lhy-20 mutant [39], we also isolated a T-DNA insertional loss-of-function mutant, which was designated lhy-7 (Fig. 1a). The lhy-7 mutant contains a single copy of T-DNA insertion in the sixth exon of $L H Y$ gene. Gene expression analysis confirmed lack of $L H Y$ transcription in the lhy-7 mutant (Fig. 1b). 


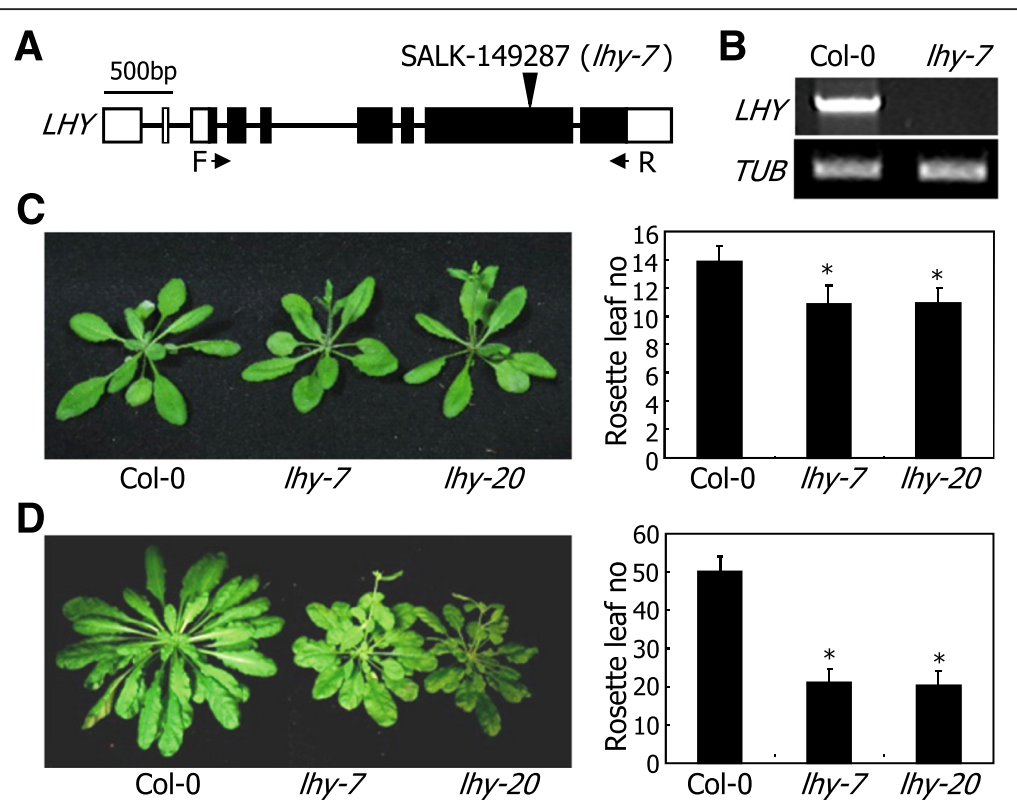

Fig. 1 LHY-defective mutants exhibit early flowering under both LDs and SDs. a Isolation of an LHY-defective mutant (Ihy- 7 ). It was isolated from a pool of T-DNA insertional lines deposited in the Arabidopsis Biological Resource Center (Ohio State University, OH). bp, base pair. F and R, forward and reverse primers, respectively, used to examine the expression of LHY gene. $\mathbf{b}$ Lack of LHY gene expression in Ihy-7 mutant. Gene expression was examined by RT-PCR. A tubulin gene (TUB) was used as control for constitutive expression. c Flowering phenotypes of Ihy mutants under LDs. The previously reported Ihy-20 mutant was also included in the assays [39]. Plants were grown until flowering in soil under LDs (16-h light and 8-h dark) (left panel). Rosette leaf numbers of 20 plants were averaged and statistically treated using Student $t$-test $\left({ }^{*} P<0.01\right)$ for each plant genotype (right pane). Bars indicate standard error of the mean (SE). d Flowering phenotypes of Ihy mutants under SDs. Plants were grown until flowering in soil under SDs (8-h light and 16-h dark) (left panel). Flowering times were measured as described in (c) (right panel)

It has been reported that the loss-of-function lhy-20 mutant exhibit a shortening of the circadian period $[39,40]$. We investigated the circadian period of the lhy-7 mutant by examining the rhythmic expression patterns of two representative clock output genes, COLD, CIRCADIAN RHYTHM, AND RNA BINDING 2 (CCR2) and CHLOROPHYLL A/B-BINDING PROTEIN 2 (CAB2) $[41,42]$. We found that the lhy-7 mutant also exhibits advanced peak expressions of the $C C R 2$ and $C A B 2$ genes compared to those observed in Col-0 plants (Additional file 1), as has been observed with other LHY-defective mutants [20, 37, 39, 40].

We next examined the flowering phenotypes of the $l h y-7$ and lhy-20 mutants under different daylengths by counting the number of rosette leaves at bolting. Both the lhy mutants showed accelerated flowering under LDs (Fig. 1c). The early flowering phenotypes of the mutants were more prominent under SDs (Fig. 1d). The reduced photoperiodic sensitivity of the lhy flowering is similar to what has been observed with LHY-defective mutants in other ecotypes $[20,37]$, showing that the altered flowering phenotypes are associated with LHY function. Since the flowering phenotypes of the $l h y-7$ and $l h y-20$ mutants were similar each other, we chose the former for subsequent molecular assays.

\section{Expression patterns of flowering genes are altered in Ihy-7 mutant}

To obtain insights into the molecular mechanism by which LHY regulates flowering time, we analyzed the expression patterns of flowering genes under LDs and SDs. In LD-grown plants, the expression of FT and SOC1 genes was slightly but detectably elevated in the $l h y-7$ mutant (Fig. 2a), which is in good agreement with the flowering phenotype of the mutant. Under SDs, the expression of FT gene, but not SOC1 gene, was markedly elevated in the lhy-7 mutant (Fig. 2b), indicating that the FT induction is the major cause of the early flowering of the mutant at least under SDs.

It is known that the circadian clock is closely associated with photoperiodic flowering $[1,17,37]$. We therefore examined the expression patterns of photoperiodic flowering genes, such as CO, GI, and FKF1, in the lhy-7 mutant. The amplitude and waveform of $C O$ transcription were not discernibly altered in the $l h y-7$ mutant under both LDs and SDs (Fig. 2a and b). In contrast, the waveforms of GI and FKF1 transcription appeared with advanced shifts of the peaks in the lhy-7 mutant under both photoperiod regimes. Considering that GI interacts with FKF1 to stabilize CO [16], it seems that the advanced expression phases of $G I$ and $F K F 1$ under SDs 


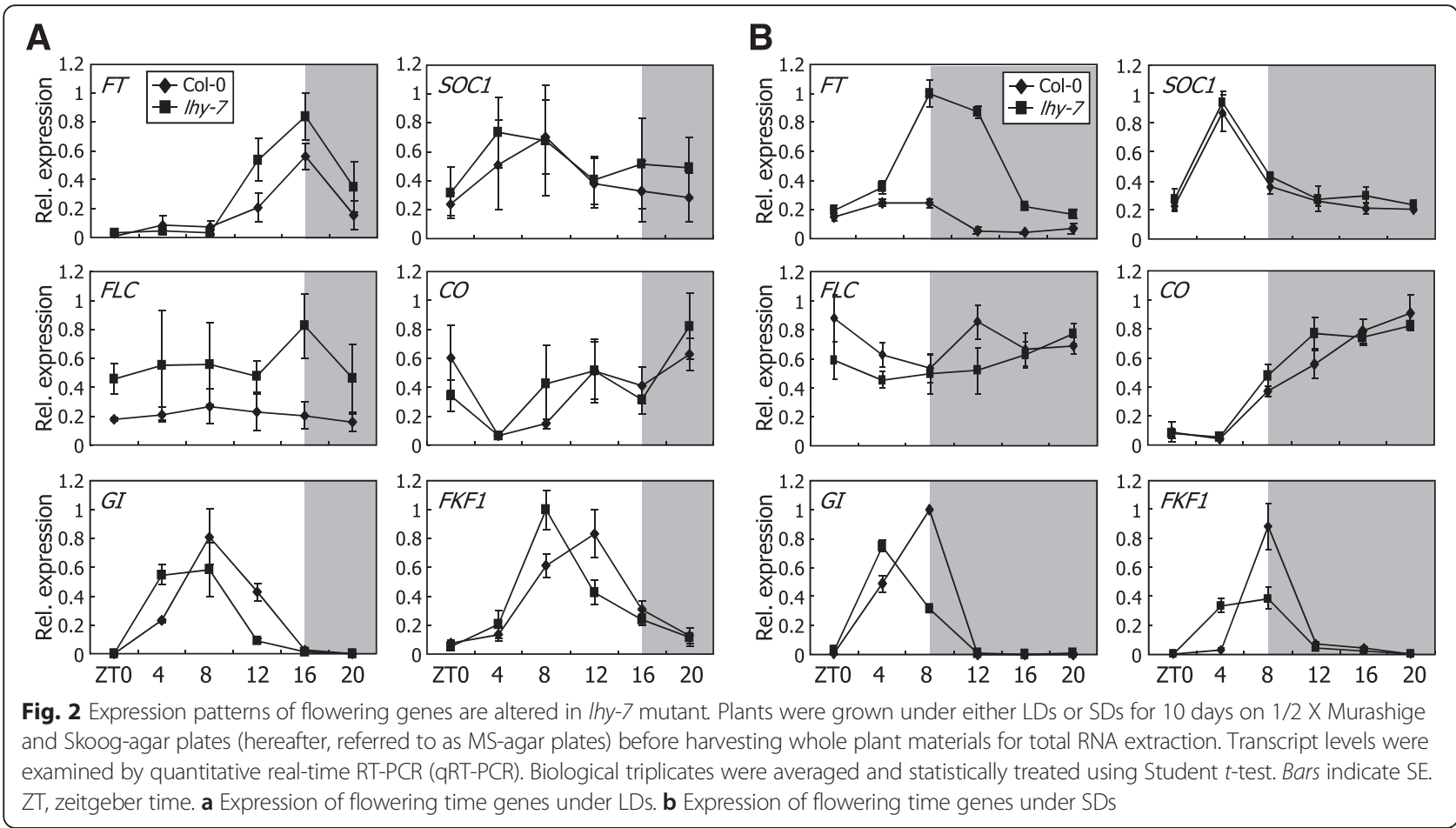

would lead to an elevation of the GI-FKF1 complex formation and stabilize $\mathrm{CO}$, underscoring the $F T$ induction and early flowering in the $l h y-7$ mutant.

We also investigated the expression patterns of flowering genes that belong to other flowering pathways, such as autonomous, thermosensory, and gibberellic acid (GA) pathways, under LDs and SDs. It was found that the expression patterns of autonomous pathway genes, such as FLOWERING LOCUS KH DOMAIN (FLK), FVE, and $F C A \gamma$, and GA pathway genes, such as SPYNDLY (SPY) and REPRESSOR OF GA1 (RGA1), were not altered in the lhy-7 mutant under both light regimes (Additional file 2). In addition, the transcription of two floral repressor genes, which play a role in temperature-responsive flowering, was not significantly affected in the mutant. While the transcript level of SHORT VEGETATIVE PHASE (SVP) gene in the mutant was comparable to that in Col-0 plants, the transcription of FLOWERING LOCUS $M \beta$ $(F L M \beta)$ gene was marginally induced in the mutant (Additional file 2). Considering the floral repressive activity of FLM $\beta$ [43], it is apparent that the early flowering phenotype of the lhy-7 mutant is not associated with the slight induction of $F L M \beta$ gene.

\section{FLC gene is not related with Ihy-7 flowering}

It has been reported that induction of the floral repressor FLOWERING LOCUS C (FLC) is linked with the late flowering phenotype of ccal lhy double mutant grown under continuous light conditions [44]. The FLC gene is also up-regulated in the parental lhy mutant in Landsberg erecta (Ler) background. We found that FLC expression is slightly increased in the lhy-7 mutant under LDs (Fig. 2a). In contrast, the $F L C$ expression was not discernibly elevated in the mutant under SDs (Fig. 2b), suggesting that $F L C$ gene is not associated with the mutant flowering phenotype.

To further examine whether $F L C$ gene is associated with the flowering phenotype of the lhy-7 mutant, we crossed the lhy-7 mutant with the FLC-defective $f l c-3$ mutant that exhibits early flowering, more prominently under SDs [45]. We compared the flowering time of the resultant lhy-7 flc-3 double mutant with those of the parental mutants. The flowering phenotype of the lhy-7 flc-3 double mutant was not discernibly different from those of the single mutants under LDs (Fig. 3a). In contrast, the $l h y-7$ flc-3 double mutant flowered earlier than the single mutants under SDs (Fig. 3b), indicating that $F L C$ gene is not directly associated with the flowering phenotype of the lhy-7 mutant.

\section{LHY does not bind to FT promoter}

LHY and CCA1 regulate a variety of genes by directly binding to the gene promoters [35, 38, 46]. For example, the CCA1 transcription factor represses SOC1 expression by binding directly to the gene promoter [38]. We found that FT gene is significantly up-regulated in the lhy-7 

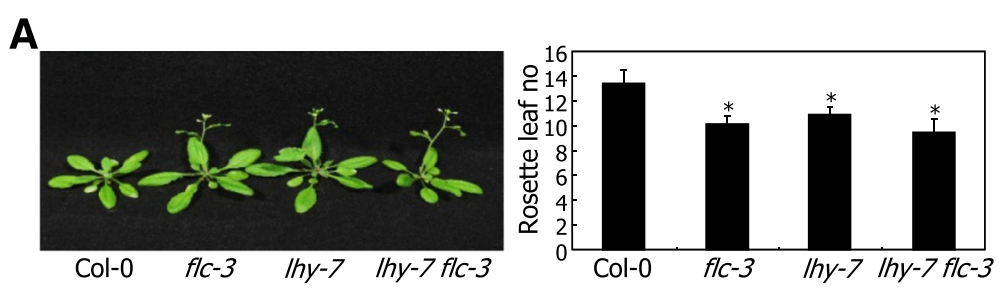

B
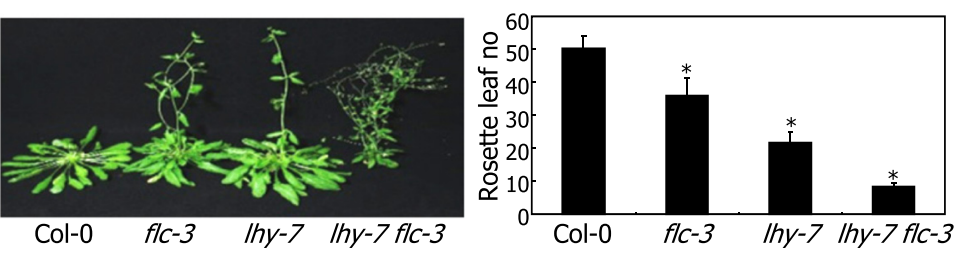

Fig. 3 FLC gene is not associated with the flowering phenotype of Ihy-7 mutant. The Ihy-7 mutant was crossed with the flc-3 mutant, resulting in Ihy-7 flc-3 double mutant. Plants were grown until flowering in soil under either LDs or SDs (left panel). Rosette leaf numbers of 20 plants were averaged and statistically treated using Student $t$-test $(* P<0.01)$ (right panel). Bars indicate SE. a Flowering phenotype of $/$ hy $-7 \mathrm{flc}-3$ double mutant under LDs. b Flowering phenotype of Ihy-7 flc-3 double mutant under SDs

mutant (Fig. 2). It was therefore suspected that LHY might repress $F T$ expression perhaps by binding to the gene promoter.

Nucleotide sequence analysis identified a putative CCA1-binding sequence (CBS) in the FT gene promoter and a potential evening element (EE) in the first intron (Fig. 4a). To examine whether LHY binds to the CBS and EE sequences, we employed chromatin immunoprecipitation (ChIP) assays using transgenic plants overexpressing a $L H Y-M Y C$ gene fusion, in which a MYC-coding sequence was fused in-frame to the $3^{\prime}$ end of the LHYcoding sequence (Additional file 3). We also included the transgenic plants overexpressing a $M Y C$-CCA1 gene fusion in the assays. Both the 35S:LHY-MYC and 35:MYC$C C A 1$ transgenic exhibited elongated hypocotyls, disruption of circadian rhythms, and suppression of FT expression (Additional file 4), as reported previously [19, 47], confirming that the transgenic plants are relevant for ChIP assays. Quantitative ChIP-PCR runs revealed that CCA1 binds to the CBS and EE sequence elements (Fig. 4b). In contrast, LHY did not bind to the sequence elements, while it efficiently bound to the TOC1 gene promoter containing EE [35].

To verify the binding of CCA1 to FT chromatin, we performed electrophoretic mobility shift assay (EMSA) using a recombinant maltose binding protein (MBP)CCA1 fusion protein. Consistent with the ChIP data, it was found that CCA1 binds specifically to CBS and EE sequence elements (Additional files 5 and 6).

To examine whether CCA1 binding to FT chromatin influences $F T$ expression, we performed transient $\beta$ glucuronidase (GUS) expression assays by coexpressing the $F T$ promoter-driven GUS reporter plasmid ( $F T_{\text {pro:- }}$ GUS) and the effector plasmids (35S:CCA1 or 35S:LHY) in Arabidopsis protoplasts. The assays showed that CCA1 negatively regulates GUS expression, but LHY does not affect GUS expression (Fig. 4c), consistent with the ChIP data. The repressive effects of CCA1 on GUS expression disappeared when a reporter plasmid harboring mutations in CBS was coexpressed, indicating that the binding of CCA1 to CBS is essential for the CCA1-mediated repression of FT expression. These observations indicate that although LHY and CCA1 are known to be functionally redundant $[20,48]$, LHY is distinct from CCA1 in regulating FT expression.

It has been reported that GI is associated with the flowering phenotype of ccal lhy double mutant [22]. While CCA1 is directly associated with GI promoter [38, 49], it is unknown whether LHY binds to GI promoter. ChIP assays using transgenic plants overexpressing a $L H Y-M Y C$ gene fusion showed that LHY also binds to GI promoter (Additional file 7), like CCA1. Binding of both LHY and CCA1 to GI promoter is in harmony with the repression of GI expression in both CCA1- and $L H Y$-inducible lines [50].

\section{Clock defects underlie the reduced photoperiodic sensitivity of lhy flowering}

LHY is a key component of the central oscillator of plant circadian clock. LHY-defective mutants exhibit a shortened circadian rhythm of approximately $20 \mathrm{~h}$ compared to that of Col-0 plants ([39], this work). We found that lhy-7 mutant exhibits early flowering with a reduced sensitivity to photoperiod. Therefore, a critical question was whether the reduced photoperiodic sensitivity of the lhy-7 flowering is interconnected with the clock defects in the mutant.

To address the question, we measured the flowering times of $l h y-7$ and lhy-20 mutants under light/dark 

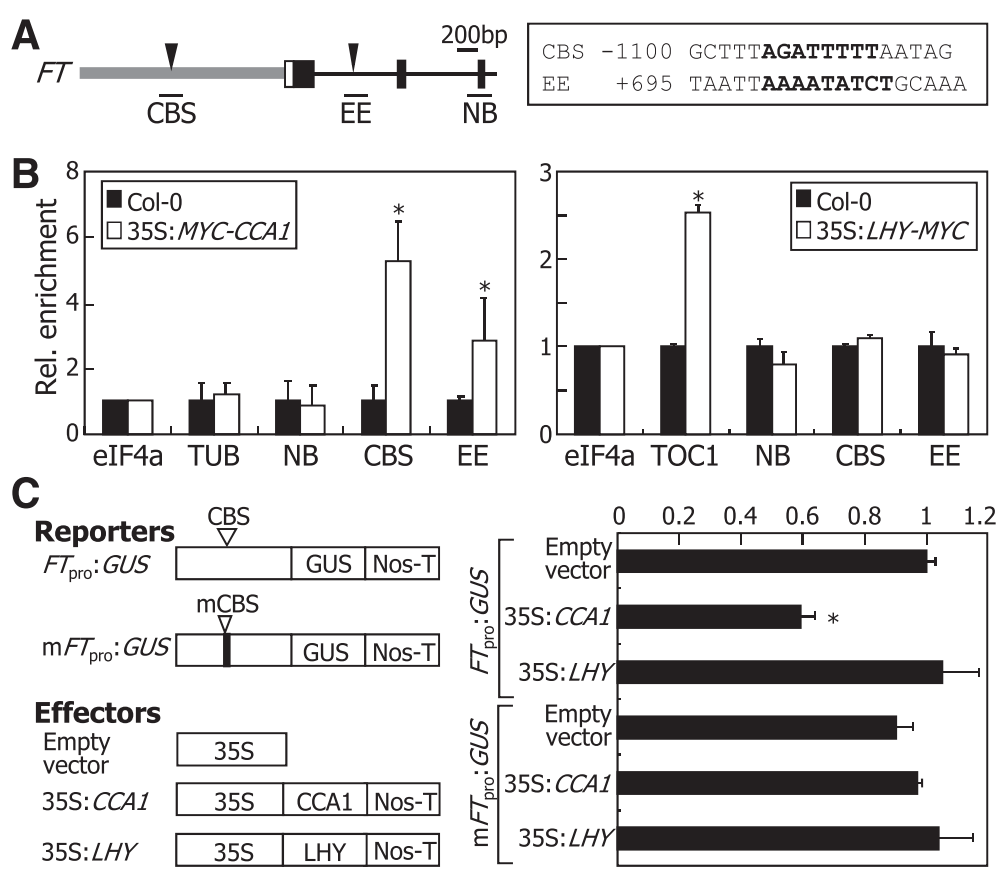

Fig. $4 \mathrm{LHY}$ does not bind to FT promoter. a Genomic structure of FT gene. (Left panel) Gray box indicates the gene promoter region. Black boxes indicate exons, and white boxes indicate untranslated regions. CBS, CCA1-binding sequence. EE, evening element. NB, non-binding sequence. (Right panel) CBS and EE sequences are listed. $\mathbf{b}$ ChIP assays on binding of CCA1 and LHY to FT promoter. A MYC-coding sequence was fused in-frame to the $5^{\prime}$ end of the CCA1-coding sequence and the $3^{\prime}$ end of the LHY-coding sequence, and the gene fusions were overexpressed driven by the Cauliflower Mosaic Virus (CaMV) 35 S promoter in Col-0 plants, resulting in 35S:MYC-CCA1 and 35S:LHY-MYC, respectively. Chromatins were prepared from 7-day-old whole plants grown on MS-agar plates and immunoprecipitated using an anti-MYC antibody. Fragmented genomic DNA was eluted from the protein-DNA complexes and subjected to quantitative PCR. Biological triplicates were averaged and statistically treated using Student $t$-test $(* P<0.01)$. Bars indicate SE. The promoter sequences of elF4a and TUB genes were included as negative controls in the assays. The promoter sequence of TIMING OF CAB EXPRESSION 1 (TOC1) gene containing EE was included as positive control [35]. c Suppression of $F /$ transcription by CCA1. The reporter and effector constructs are illustrated (left panel). Transient GUS expression assays were performed using Arabidopsis protoplasts (right panel). Five measurements were averaged and statistically treated (t-test, $\left.{ }^{*} P<0.01\right)$. Bars indicate SE

(L/D) cycles that were matched to the endogenous circadian periods of the mutants. If the altered flowering times of the lhy mutants are entirely due to the clock defects, the flowering times would be restored by matching the external $\mathrm{L} / \mathrm{D}$ cycles to the endogenous circadian period, as has been observed with TOC1-defective mutants [21, 28]. As expected, we found that the early flowering of the lhy mutants was completely annulled under SDs of $20 \mathrm{~h}$ (6.7 L: 13.3D) (Fig. 5a), which matches to the endogenous period of the mutants ([39], this work), indicating that LHY regulates flowering time entirely via the circadian clock.

We also analyzed the expression profiles of flowering time genes in the lhy-7 mutant under SDs of adjusted L/D cycles. The elevation of $F T$ transcript levels was not observed when the lhy-7 mutant was grown under SDs of $20 \mathrm{~h}$ (Fig. 5b and Additional file 8), as has been observed with short-period plants, such as toc1 null mutants $[28,29]$. It is therefore evident that the reduced photoperiod sensitivity of the $l h y-7$ flowering is caused by the clock defects.
It was found that $C O$ transcription was elevated at night under the assay conditions (Fig. 5b). Since $\mathrm{CO}$ protein is degraded under dark conditions [13, 14], it is unlikely that the $C O$ induction at night is physiologically important for the flowering phenotype of the lhy-7 mutant. The waveform of GI transcription under the adjusted L/D cycles was comparable to that in Col-0 plants under SDs of $24 \mathrm{~h}$. In addition, gene expression assays revealed that the expression of flowering genes, such as $F L K, F V E, F C A \gamma, S V P, S P Y$, and RGA1, was not altered in the lhy-7 mutant under both SDs of 20 and $24 \mathrm{~h}$. The slight induction of FLM $\beta$ gene in the lhy-7 mutant under SDs of $24 \mathrm{~h}$ was also observed under SDs of $20 \mathrm{~h}$ (Additional files 2 and 9). On the basis of the flowering times and expression patterns of flowering genes in the lhy mutants under adjusted L/D cycles, we concluded that LHY regulates photoperiodic flowering via the clock function.

To verify that LHY regulates flowering time through the clock-controlled CO-FT pathway, we generated lhy-7 co-101 double mutant by crossing the lhy-7 mutant 

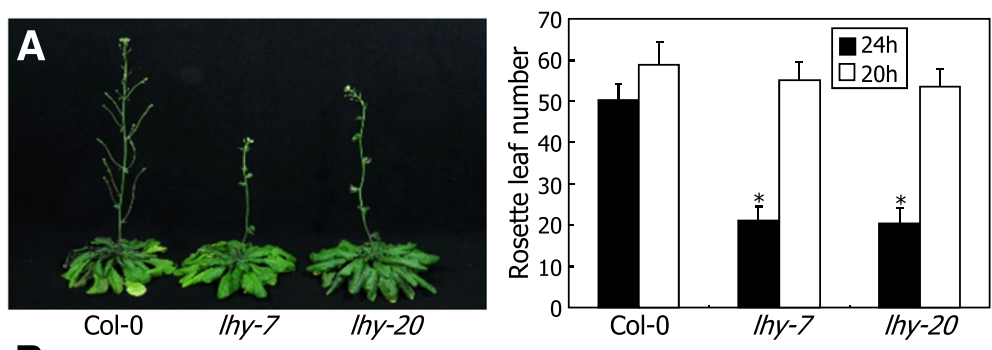

B
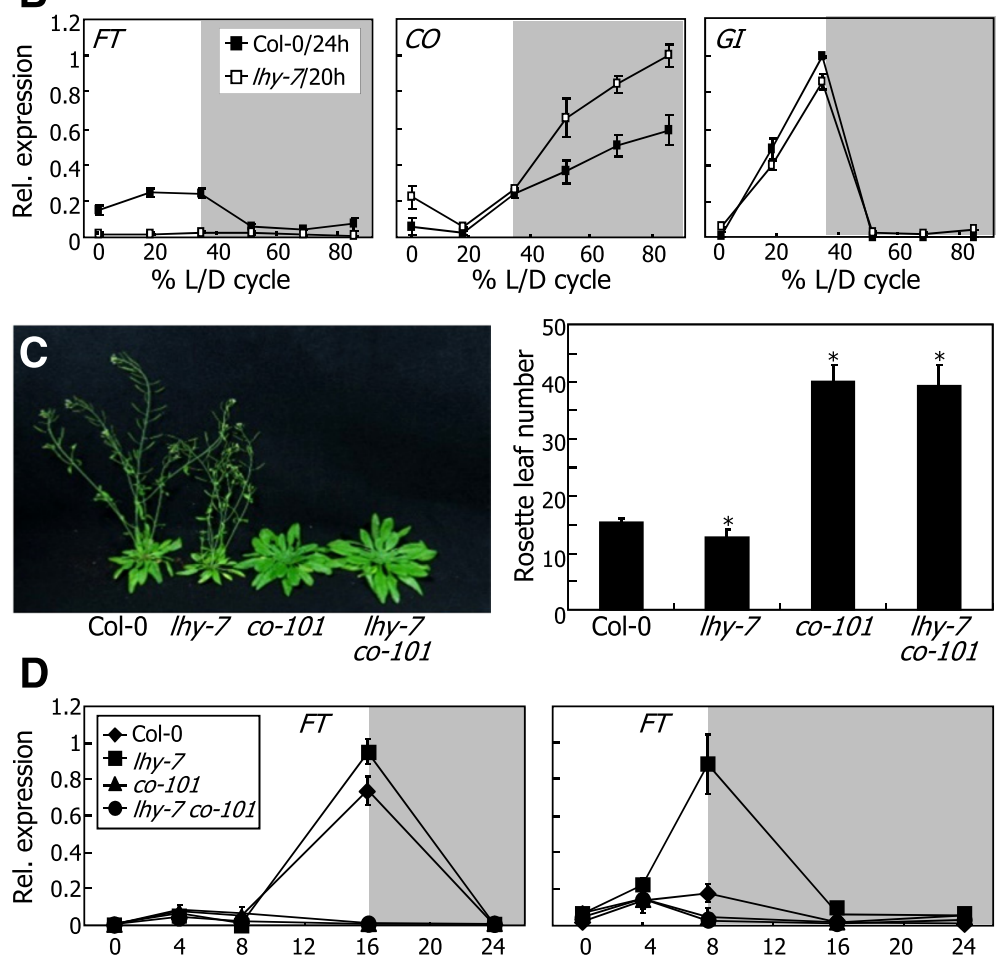

Fig. 5 Clock defects underlie the reduced photoperiodic sensitivity of Ihy flowering. a Flowering times of Ihy mutants under SDs of differential total duration. Plants were grown until flowering under SDs of 24-h or 20-h total duration. Those grown under SDs of 20-h total duration were photographed (left panel). Rosette leaf numbers of 20 plants were averaged and statistically treated ( $t$-test, $\left.{ }^{*} P<0.01\right)$ (right panel). Bars indicate SE. b Expression profiles of $F T$ and clock output genes in Ihy-7 mutant grown under SDs of 24-h or 20-h total duration. Transcript levels were examined by qRT-PCR. L/D, light/dark. c Flowering phenotype of Ihy-7 co-101 double mutant under LDs. The Ihy-7 mutant was crossed with the co-101 mutant, resulting in Ihy-7 co-101 double mutant. Plants were grown until flowering in soil under LDs (left panel). Flowering times were measured as described in (a) (right panel). $\mathbf{d}$ Expression of FT gene in Ihy-7 co-101 double mutant grown under either LDs or SDs. Transcript levels were examined by qRT-PCR. Biological triplicates were averaged. Bars indicate SE

with the CO-defective co-101 mutant. Acceleration of flowering by the lhy mutation completely disappeared in the lhy-7 co-101 double mutant under LDs (Fig. 5c). Accordingly, FT expression was slightly induced in the lhy-7 mutant, but the induction was compromised in the lhy-7 co-101 double mutant (Fig. 5d). We also examined the level of $F T$ transcripts in the single and double mutants under SDs, since the early flowering phenotype of the single mutant was more prominent under this light regime (Fig. 1d). We found that the elevation of FT expression in the lhy-7 mutant was completely annulled in the lhy-7 co-101 double mutant (Fig. 5d). Together, these observations indicate that LHY-mediated regulation of photoperiodic flowering depends on $\mathrm{CO}$ function.

\section{Discussion}

LHY-mediated clock control of photoperiodic flowering Plants sense photoperiodic changes by integrating light signals perceived by the photoreceptors and timing information provided by the circadian clock. In Arabidopsis, photoperiod-sensitive induction of the floral integrator $F T$ is a crucial molecular event in photoperiodic flowering [6]. It is known that the coordinated action of light signals and timing information allows the $\mathrm{CO}$ transcription factor to accumulate specifically in late afternoon under LDs, 
which is a prerequisite for the LD-specific induction of $F T$ gene $[1-3,12]$. The circadian clock regulates $\mathrm{CO}$ activity at both the transcriptional and posttranscriptional levels $[12,16,23]$. At the transcriptional level, the clock shapes the rhythmic expression patterns of $C O$ in a way that a high level of $C O$ transcripts accumulates during the light phase under LDs [23]. At the posttranscriptional level, the circadian clock contributes to the stabilization of $\mathrm{CO}$ in late afternoon under LDs by modulating the expression of GI and $F K F 1$ genes $[16,17]$.

In this study, we demonstrated that LHY, which is a core component of the central oscillator in Arabidopsis, regulates photoperiodic flowering by adjusting the rhythmic expression patterns of photoperiodic flowering genes, such as $G I$ and $F K F 1$. We found that the expression peaks of GI and FKF1 genes are shifted earlier in the lhy-7 mutant, which is consistent with the shortened circadian period of the mutant. A plausible explanation is that the advanced phases of GI and FKF1 expression in the lhy-7 mutant would lead to an increase in the formation of GI-FKF1 complexes during the light phase, resulting in a higher-level accumulation of $\mathrm{CO}$ in the mutant. In support of this view, the early flowering phenotype of the mutant was completely annulled by matching the external L/D cycles to the endogenous circadian period. Under these assay conditions, the phase shift of GI transcription was restored and the up-regulated expression of $F T$ was suppressed to a basal level in the lhy-7 mutant. Together with the previous reports on shortperiod plants $[28,29]$, it seems that the circadian clock components, including LHY, regulates photoperiodic flowering by adjusting the expression timing of photoperiodic flowering genes.

\section{Common and distinct roles of LHY and CCA1 in photoperiodic flowering}

LHY and CCA1 are MYB motif-containing transcription factors that function at least in part redundantly in the circadian clock [19, 20,48]. Whereas the gain-of-function mutations of both $L H Y$ and $C C A 1$ genes disrupt circadian rhythms $[19,20]$, both the LHY- and CCA1-defective mutants exhibit shortened circadian periods $[20,37,51]$. The ccal lhy double mutants show shorter circadian periods than the ccal or lhy single mutants [20,37, 48].

On the other hand, there have been some reports supporting distinct roles of CCA1 and LHY. For instance, $L H Y$ overexpression does not enhance pathogen resistance, whereas $C C A 1$ overexpression induces pathogen resistance [52, 53]. Another example is the differential regulation of CCA1 and $L H Y$ expression by CCA1 HIKING EXPEDITION (CHE) and BROTHER OF LUX ARRHYTHMON (BOA), the components of the Arabidopsis circadian clock. While CHE and BOA bind directly to $C C A 1$ gene promoter, they are not associated with $L H Y$ gene promoter $[46,54]$.

We found that CCA1, but not LHY, binds to FT gene promoter to repress its expression. It has been reported that CCA1 represses $S O C 1$ expression by binding to the gene promoter [38]. We observed that SOC1 expression is not discernibly affected by lhy mutations, suggesting that LHY is not related with SOC1 expression. It is known that LHY and CCA1 form both homodimers and heterodimers in vivo [47, 55]. One possibility is that whereas common roles of the two transcription factors would be related with the LHY-CCA1 heterodimers, their distinct roles would be exerted through the homodimers.

\section{Clock-independent control of photoperiodic flowering}

It is now apparent that most clock components, including LHY, TOC1, and CKB4, regulate photoperiodic flowering via the clock function $[28,29]$. Notably, the shorter the circadian periods of clock mutants, the earlier the flowering times of the mutants in most cases [37], supporting that the clock regulates photoperiodic flowering by modulating the rhythmic expression of photoperiodic flowering genes $[1,6,17]$.

However, there are recent reports supporting that some clock components affect flowering time through clock-independent pathways. CCA1 regulates the expression of floral integrators by directly binding to the gene promoters ([38], this work). GI also binds directly to $F T$ gene promoter [30]. In addition, the early flowering phenotype of DET1-defective mutants is not restored by matching the external L/D cycles to the endogenous circadian period of the mutants, indicating that DET1 negatively regulates flowering independent of its role in the clock function [32]. Together, it is likely that clock components regulate photoperiodic flowering through both $\mathrm{CO}$-mediated, clock-dependent and $\mathrm{CO}$-free, clockindependent pathways. It will be interesting to investigate how individual clock components regulate flowering time and how the clock-dependent and clock-independent pathways are functionally inter-connected with each other in photoperiodic flowering.

\section{Conclusions}

We investigated how LHY regulates photoperiodic flowering by performing molecular genetic and biochemical studies. LHY regulates photoperiodic flowering entirely via the circadian clock. In the LHY-defective mutants, the early flowering phenotypes and the shifted phases of photoperiodic flowering gene expression were recovered by matching the external $\mathrm{L} / \mathrm{D}$ cycles to the endogenous circadian periods of the mutants. It is notable that the mechanism by which LHY regulates photoperiodic flowering is somewhat distinct from that exerted by CCA1. 
Our findings would contribute to better understanding of how the clock function is associated with flowering time control in response to photoperiodic signals.

\section{Methods}

Plant genotypes and growth conditions

Arabidopsis thaliana lines used were in the Columbia (Col-0) background, unless specified otherwise. Arabidopsis plants were grown either in soil or on 1/2 X Murashige and Skoog (MS)-agar plates (hereafter referred to as MS-agar plates) at $23{ }^{\circ} \mathrm{C}$ under either LDs (16-h light and 8-h dark) or SDs of $24 \mathrm{~h}(8 \mathrm{~L}: 16 \mathrm{D})$ or $20 \mathrm{~h}(6.7 \mathrm{L:}: 13.3 \mathrm{D})$ total duration. White light illumination $\left(120 \mu \mathrm{mol}\right.$ photons $\left.\mathrm{m}^{-2} \mathrm{~s}^{-1}\right)$ was provided by fluorescent FLR40D/A tubes (Osram, Seoul, Korea).

T-DNA insertional gene knockout mutants lhy-20, flc-3, and $c o-101$ have been described previously $[39,45,56]$. The $L H Y$-deficient lhy-7 mutant (SALK-149287) was isolated from a T-DNA insertional mutant pool deposited in the Arabidopsis Information Resource (TAIR, Ohio State University, OH). Homozygotic lines were obtained by selection for three or more generations and analysis of segregation ratios. Lack of gene expression in the mutants was verified by RT-PCR before use.

A MYC-coding sequence was fused in-frame to the $5^{\prime}$ end of the CCA1-coding sequence or to the $3^{\prime}$ end of the LHY-coding sequence, and the gene fusions were subcloned into the pBA002 vector under the control of the Cauliflower Mosaic Virus (CaMV) 35S promoter. The expression constructs were transformed into Col-0 plants, resulting in 35S:MYC-CCA1 and 35S:LHY-MYC, respectively. Overexpression of the transgenes was verified by quantitative real-time RT-PCR (qRT-PCR).

\section{Gene expression assay}

Extraction of total RNA samples from appropriate plant materials and qRT-PCR conditions have been described previously [57]. Total RNA samples were pretreated with RNase-free DNase to eliminate contaminating genomic DNA before use.

RNA sample preparation, reverse transcription, and quantitative PCR were conducted according to the criteria that have been proposed to ensure reproducible and accurate measurements [58].

qRT-PCR reactions were performed in 96-well blocks with an Applied Biosystems 7500 Real-Time PCR System (Foster City, CA) using the SYBR Green I master mix in a reaction volume of $20 \mu \mathrm{l}$. The PCR primers were designed using the Primer Express Software installed in the system and listed in Additional file 10. The two-step thermal cycling profile employed was $15 \mathrm{~s}$ at $94{ }^{\circ} \mathrm{C}$ and $1 \mathrm{~min}$ at $68^{\circ} \mathrm{C}$. An eIF4A gene (At3g13920) was included in the reactions as internal control to normalize the variations in the amounts of cDNA used. All the qRT-PCR reactions were performed in biological triplicates using RNA samples extracted from three independent plant materials grown under identical conditions. The comparative $\Delta \Delta C_{T}$ method was employed to evaluate relative quantities of each amplified product in the samples. The threshold cycle $\left(C_{T}\right)$ was automatically determined for each reaction using the default parameters of the system. The specificity of PCR reactions was determined by melt curve analysis of the amplified products using the standard method installed in the system.

\section{ChIP assay}

ChIP assays were performed, essentially as described previously [59], in biological triplicates using three independent plant materials grown under identical conditions. Sevenday-old 35S:MYC-CCA1 and 35S:LHY-MYC transgenic plants grown on MS-agar plates were vacuum-infiltrated with $1 \%(\mathrm{v} / \mathrm{v})$ formaldehyde for cross-linking and ground in liquid nitrogen after quenching the cross-linking process. Chromatin preparations were sonicated into 0.5to 1-kb fragments. An anti-MYC antibody (Millipore, Billerica, MA) was added to the chromatin solution, which was precleared with salmon sperm DNA/protein G agarose beads (Roche, Indianapolis, IN). The precipitates were eluted from the beads. Cross-links were reversed, and residual proteins were removed by incubation with proteinase K. DNA was recovered using the QIAquick PCR purification kit (Qiagen, Valencia, CA). Quantitative PCR was performed to determine the amounts of genomic DNA enriched in the chromatin preparations. The primers used are listed in Additional file 10.

\section{Transient expression assays in Arabidopsis protoplasts}

In the reporter vector, a $2-\mathrm{kb}$ promoter sequence of $F T$ gene was transcriptionally fused to the $\beta$-glucuronidase (GUS) gene. The GUS reporter construct harboring a mutated CCA1-binding sequence (CBS) within the FT promoter was used to investigate the effects of CBS on the binding of CCA1 and LHY to the FT promoter. The CCA1- and LHY-coding sequences were subcloned under the control of the CaMV $35 \mathrm{~S}$ promoter in the effector vector. The reporter and effector vectors were cotransfected into Arabidopsis mesophyll protoplasts by the polyethylene glycol (PEG)-calcium transfection method [60]. The CaMV $35 \mathrm{~S}$ promoter-luciferase construct was also cotransfected as internal control. GUS activity was measured by a fluorometric method as described previously [61]. Luciferase activity assay was performed using the Luciferase Assay System kit (Promega, Madison, WI). GUS activities were normalized by luciferase activities.

\section{Flowering time measurement}

Plants were grown in soil at $23{ }^{\circ} \mathrm{C}$ under either long days of $24 \mathrm{~h}$ or short days of $24 \mathrm{~h}(8 \mathrm{~L}: 16 \mathrm{D})$ or $20 \mathrm{~h}$ (6.6 L: 
13.4D) total duration until flowering. Numbers of rosette leaves at bolting were counted, and 20 countings were averaged for each measurement.

\section{Circadian rhythm measurement}

Plants were entrained to long day cycles and then transferred to continuous light conditions for 3 days. To trace the circadian rhythm, whole plant materials were harvested at appropriate zeitgeber time (ZT) points for total RNA extraction. Gene transcript levels were measured by qRT-PCR.

\section{Preparation of recombinant MBP-CCA1 fusion protein}

A CCA1-coding sequence was subcloned into the pMAL-c2X Escherichia coli (E. coli) expression vector (NEB, Ipswich, MA) harboring a maltose binding protein (MBP)-coding sequence. Recombinant MBP-CCA1 fusion protein was produced in E. coli Rosetta2 (DE3) pLysS strain (Novagen, Madison, WI). Harvested cells were resuspended in MBP buffer $(20 \mathrm{mM}$ Tris- $\mathrm{HCl}, \mathrm{pH} 7.4,200 \mathrm{mM} \mathrm{NaCl}$, $1 \mathrm{mM}$ EDTA, $10 \mathrm{mM}$ 2-mercaptoethanol, $1 \mathrm{mM}$ PMSF, and protease inhibitor cocktail (Roche, Indianapolis, IN)). Cell lysates were prepared by running three cycles of freezing and thawing followed by centrifugation. The fusion proteins were affinity-purified as described previously [62].

\section{EMSA}

EMSA was performed using recombinant MBP-CCA1 fusion protein, as described previously [63]. DNA fragments were end-labeled with $\gamma^{-}{ }^{32} \mathrm{P}[\mathrm{dATP}]$ using T4 polynucleotide kinase (Takara, Kyoto, Japan). Labeled probes were incubated with $100 \mathrm{ng}$ of MBP or MBP-CCA1 fusion protein for $30 \mathrm{~min}$ at room temperature in binding buffer (10 mM Tris- $\mathrm{HCl}, \mathrm{pH} 7.6,50 \mathrm{mM} \mathrm{NaCl}, 1 \mathrm{mM}$ EDTA, $5 \mathrm{mM}$ DTT, $5 \%$ glycerol) supplemented with $100 \mathrm{ng}$ poly $(\mathrm{dI}-\mathrm{dC})$ in the presence or absence of competitor DNA fragments. The reaction mixtures were resolved on $6 \%$ non-denaturing polyacrylamide gel at $100 \mathrm{~V}$ for $1 \mathrm{~h}$. The gels were dried on Whatman $3 \mathrm{MM}$ paper and exposed to X-ray film.

\section{Additional files}

Additional file 1: Circadian rhythms in LHY-defective mutants. Ten-day-old plants grown on $1 / 2 \times$ Murashige and Skoog-agar plates (hereafter, referred to as MS-agar plates) under long days (LDs, 16-h light and 8-h dark) were transferred to continuous light conditions at dawn (upper diagram). DAC, days after cold imbibition. Whole plants materials were harvested at the indicated zeitgeber time (ZT) points for total RNA extraction (lower panel). Rhythmic expression of CHLOROPHYLL A/B-BINDING PROTEIN 2 (CAB2) and CAROTENOID AND CHLOROPLAST REGULATION 2 (CCR2) genes, which exhibit circadian rhythmic expression patterns [41, 42], was examined by quantitative real-time RT-PCR (qRT-PCR). Biological triplicates were averaged. Bars indicate standard error of the mean. Two LHY-defective mutants (Ihy-7 and Ihy-20) were examined. (PDF $157 \mathrm{~kb}$ )
Additional file 2: Expression of flowering time genes in Ihy-7 mutant. Plants were grown under either LDs or short days (SDs, 8-h light and 16-h dark) for 10 days on MS-agar plates. Whole plants were harvested at ZT 8 for total RNA extraction. Transcript levels were examined by qRT-PCR. Biological triplicates were averaged and statistically treated using Student $t$-test ( ${ }^{*} P<0.01$ ). Bars indicate standard error of the mean. (PDF $131 \mathrm{~kb}$ )

Additional file 3: Levels of $L H Y$ and CCA1 transcripts in 35S:LHY-MYC and 35S:MYC-CCA1 transgenic plants, respectively. Ten-day-old whole plants grown on MS-agar plates under LDs were harvested for total RNA extraction at the indicated ZT points. Transcript levels were examined by qRT-PCR. Biological triplicates were averaged and statistically treated ( $t$-test, $\left.{ }^{*} P<0.01\right)$. Bars indicate standard error of the mean. (PDF $121 \mathrm{~kb}$ )

Additional file 4: Functionality of 35S:MYC-CCA1 and 35S:LHY-MYC transgenic plants. A and B. Elongated hypocotyls. Plants were grown on MS-agar plates for 5 days under either LDs (A) or SDs (B). Measurements of 20 seedlings were averaged and statistically treated ( $t$-test, $\left.{ }^{*} P<0.01\right)$. Bars indicate standard error of the mean. C. Disruption of circadian rhythms. Expression patterns of CCR2 gene were examined as described in Additional file 1. Bars indicate standard error of the mean. D. Suppression of FT transcription. Plants were grown under LDs for 10 days on MS-agar plates. Whole plants were harvested at ZT16 for total RNA extraction. Transcript levels were examined as described in Additional file 2. Bars indicate standard error of the mean. (PDF $195 \mathrm{~kb}$ )

Additional file 5: Recombinant proteins used for electrophoretic mobility shift assay (EMSA). Recombinant maltose-binding protein (MBP) and MBPCCA1 fusion protein were prepared in E. coli cells and affinity-purified. Protein quality was verified by running on $10 \%$ SDS-PAGE and Coomassie brilliant blue staining. The arrow and arrowhead indicate full-size MBP and MBP-CCA1 proteins, respectively. SM, size marker. kDa, kilodalton. (PDF 263 kb)

Additional file 6: EMSA on binding of MBP and MBP-CCA1 proteins to conserved sequences in FT locus. Recombinant MBP and MBP-CCA1 fusion proteins were prepared as described in Additional file 5. Radio-labelled CCA1binding sequence (CBS) and evening element (EE) DNA fragments, which were described in Fig. 4a, were used. A. EMSA on MBP binding to DNA fragments. $(+)$ and $(-)$ indicate assays with or without MBP protein. Note that $\mathrm{MBP}$ alone does not bind to the CBS and EE sequences. B. EMSA on MBPCCA1 binding to DNA fragments. The core sequences of CBS and EE were mutated, resulting in $\mathrm{mCBS}$ and $\mathrm{mEE}$, respectively. Excess amounts (50X,100X) of unlabeled DNA fragments were added as competitors. (PDF $182 \mathrm{~kb}$ )

Additional file 7: ChIP assays on LHY binding to G/ promoter. Chromatins were prepared from 7-day-old whole plants grown on MS-agar plates and immunoprecipitated using an anti-MYC antibody. Fragmented genomic DNA was eluted from the protein-DNA complexes and subjected to quantitative PCR. Biological triplicates were averaged and statistically treated using Student t-test ( $\left.{ }^{*} P<0.01\right)$. Bars indicate standard error of the mean. GI (NB) amplifies a downstream sequence region of $G$ l gene, and $G$ (CBS) amplifies a sequence region containing CBS in the $\mathrm{Gl}$ promoter, which has been described previously [38]. (PDF $122 \mathrm{~kb}$ )

Additional file 8: Expression of flowering genes in Ihy-7 mutant under short days of 20-h total duration. Plants were grown for 10 days under short-day cycles of either 24-h (8-h light and 16-h dark) or 20-h (6.7-h light and 13.3-h dark) total duration. Whole plant materials were harvested throughout the \% light-dark (L/D) cycles. Transcript levels were examined by qRT-PCR in Col-0 plants (A) and /hy-7 mutant (B). Biological triplicates were averaged. Bars indicate standard error of the mean. h, hour. (PDF $133 \mathrm{~kb}$ )

Additional file 9: Expression of flowering genes in Ihy-7 mutant under SDs of 20-h total duration. Plants were grown for 10 days under short-day cycles of 20-h total duration (6.7-h light and 13.3-h dark). Whole plant materials were harvested for total RNA extraction. Transcript levels were examined by qRTPCR. Biological triplicates were averaged and statistically treated using Student $t$-test ( $\left.{ }^{*} P<0.01\right)$. Bars indicate standard error of the mean. (PDF $130 \mathrm{~kb}$ )

Additional file 10: Primers used in $q R T-P C R, R T-P C R$, and ChIP-qPCR. F, forward primer; $\mathrm{R}$, reverse primer. (PDF $90 \mathrm{~kb}$ )

\section{Abbreviations}

BOA: BROTHER OF LUX ARRHYTHMO; CAB2: CHLOROPHYLL A/B-BINDING PROTEIN 2; CBS: CCA1-binding sequence; CCA1: CIRCADIAN CLOCK ASSOCIATED 1; CCR2: COLD, CIRCADIAN RHYTHM, AND RNA BINDING 2; 
CDF1: CYCLING DOF FACTOR 1; CHE: CCA1 HIKING EXPEDITION; ChIP: chromatin immunoprecipitation; CKB4: CASEIN KINASE II BETA SUBUNIT 4; CO: CONSTANS; COP1: CONSTITUTIVE PHOTOMORPHOGENIC 1; DET1: DEETIOLATED 1; EE: evening element; EMSA: electrophoretic mobility shift assay; FKF1: FLAVIN-BINDING, KELCH REPEAT, F-BOX PROTEIN 1; FLC: FLOWERING LOCUS C; FLK: FLOWERING LOCUS KH DOMAIN; FLM: FLOWERING LOCUS M; FT: FLOWERING LOCUS T; GA: gibberellic acid; GI: GIGANTEA; GUS: $\beta$ glucuronidase; HOS1: HIGH EXPRESSION OF OSMOTICALLY RESPONSIVE GENES 1; LHY: LATE ELONGATED HYPOCOTYL; L/D: light/dark; LD: long day; MBP: maltose binding protein; RGA1: REPRESSOR OF GA1; SD: short day; SOC1: SUPPRESSOR OF CONSTANS OVEREXPRESSION 1; SPY: SPINDLY; SVP: SHORT VEGETATIVE PAHSE; TOC1: TIMING OF CAB EXPRSSION 1; YFP: yellow fluorescence protein.

\section{Acknowledgements}

This work was supported by the Leaping Research (NRF-2015R1A2A1A05001636) and Global Research Lab (NRF-2012K1A1A2055546) Programs provided by the National Research Foundation of Korea and the Next-Generation BioGreen 21 Program (PJ0111532015) provided by the Rural Development Administration of Korea. KEG was partially supported by Global PH.D Fellowship Program through the National Research Foundation of Korea (NRF-2015H1A2A1034250).

\section{Availability of supporting data}

The data sets supporting the results of this article are included within the article and its additional files.

\section{Authors' contributions}

CMP conceived and designed the project. MJP and YJK performed the molecular and biochemical assays. YJK and KEG measured flowering time of the mutants. CMP, MJP, YJK analyzed the data. CMP and MJP wrote the manuscript. All authors discussed the results and approved the final form of the manuscript.

\section{Competing interests}

The authors declare that they have no competing interests.

\section{Consent to publish}

Not applicable.

\section{Ethics}

Not applicable.

\section{Received: 29 February 2016 Accepted: 17 May 2016} Published online: 20 May 2016

\section{References}

1. Mouradov A, Cremer F, Coupland G. Control of flowering time: interacting pathways as a basis for diversity. Plant Cell. 2002;14:S111-30.

2. Amasino R. Seasonal and developmental timing of flowering. Plant J. 2010; 61(6):1001-13.

3. Andrés $F$, Coupland $G$. The genetic basis of flowering responses to seasonal cues. Nat Rev Genet. 2012;13(9):627-39.

4. Moon J, Lee H, Kim M, Lee I. Analysis of flowering pathway integrators in Arabidopsis. Plant Cell Physiol. 2005;46(2):292-9.

5. Corbesier $\mathrm{L}$, Coupland $\mathrm{G}$. The quest for florigen: a review of recent progress. J Exp Bot. 2006:57(13):3395-403.

6. Song $\mathrm{YH}$, Ito $\mathrm{S}$, Imaizumi T. Flowering time regulation: photoperiod- and temperature-sensing in leaves. Trends Plant Sci. 2013;18(10):575-83.

7. Robson F, Costa MM, Hepworth SR, Vizir I, Piñeiro M, Reeves PH, et al. Functional importance of conserved domains in the flowering-time gene CONSTANS demonstrated by analysis of mutant alleles and transgenic plants. Plant J. 2001;28(6):619-31.

8. An H, Roussot C, Suárez-López P, Corbesier L, Vincent C, Piñeiro M, et al. CONSTANS acts in the phloem to regulate a systemic signal that induces photoperiodic flowering of Arabidopsis. Development. 2004;131(15):3615-26.

9. Yoo SK, Chung KS, Kim J, Lee JH, Hong SM, Yoo SJ, et al. CONSTANS activates SUPPRESSOR OF OVEREXPRESSION OF CONSTANS 1 through FLOWERING LOCUS T to promote flowering in Arabidopsis. Plant Physiol. 2005:139(2):770-8

10. Putterill J, Robson F, Lee K, Simon R, Coupland G. The CONSTANS gene of Arabidopsis promotes flowering and encodes a protein showing similarities to zinc finger transcription factors. Cell. 1995;80(6):847-57.
11. Samach A, Onouchi H, Gold SE, Ditta GS, Schwarz-Sommer Z, Yanofsky MF, et al. Distinct roles of CONSTANS target genes in reproductive development of Arabidopsis. Science. 2000;288(5471):1613-6.

12. Valverde F, Mouradov A, Soppe W, Ravenscroft D, Samach A, Coupland G. Photoreceptor regulation of CONSTANS protein in photoperiodic flowering. Science. 2004:303(5660):1003-6.

13. Jang S, Marchal V, Panigrahi KC, Wenkel S, Soppe W, Deng XW, et al. Arabidopsis COP1 shapes the temporal pattern of $\mathrm{CO}$ accumulation conferring a photoperiodic flowering response. EMBO J. 2008;27(8):1277-88.

14. Liu LJ, Zhang YC, Li QH, Sang Y, Mao J, Lian HL, et al. COP1-mediated ubiquitination of CONSTANS is implicated in cryptochrome regulation of flowering in Arabidopsis. Plant Cell. 2008;20(2):292-306.

15. Lazaro A, Valverde F, Piñeiro M, Jarillo JA. The Arabidopsis E3 ubiquitin ligase HOS1 negatively regulates CONSTANS abundance in the photoperiodic control of flowering. Plant Cell. 2012;24(3):982-99.

16. Song YH, Smith RW, To BJ, Millar AJ, Imaizumi T. FKF1 conveys timing information for CONSTANS stabilization in photoperiodic flowering. Science. 2012;336(6084):1045-9.

17. Song YH, Shim JS, Kinmonth-Schultz HA, Imaizumi T. Photoperiodic flowering: time measurement mechanisms in leaves. Annu Rev Plant Biol. 2015;66:441-64.

18. Hicks KA, Millar AJ, Carré IA, Somers DE, Straume M, Meeks-Wagner DR, et al. Conditional circadian dysfunction of the Arabidopsis early-flowering 3 mutant. Science. 1996:274(5288):790-2.

19. Wang ZY, Tobin EM. Constitutive expression of the CIRCADIAN CLOCK ASSOCIATED 1 (CCA1) gene disrupts circadian rhythms and suppresses its own expression. Cell. 1998;93(7):1207-17.

20. Mizoguchi T, Wheatley K, Hanzawa Y, Wright L, Mizoguchi M, Song HR, et al. LHY and CCA1 are partially redundant genes required to maintain circadian rhythms in Arabidopsis. Dev Cell. 2002;2(5):629-41.

21. Strayer C, Oyama T, Schultz TF, Raman R, Somers DE, Más P, et al. Cloning of the Arabidopsis clock gene TOC1, an autoregulatory response regulator homolog. Science. 2000;289(5480):768-71.

22. Mizoguchi T, Wright L, Fujiwara S, Cremer F, Lee K, Onouchi H, et al. Distinct roles of GIGANTEA in promoting flowering and regulating circadian rhythms in Arabidopsis. Plant Cell. 2005;17(8):2255-70.

23. Suárez-López P, Wheatley K, Robson F, Onouchi H, Valverde F, Coupland G. CONSTANS mediates between the circadian clock and the control of flowering in Arabidopsis. Nature. 2001:410(6832):1116-20.

24. Nelson DC, Lasswell J, Rogg LE, Cohen MA, Bartel B. FKF1, a clock-controlled gene that regulates the transition to flowering in Arabidopsis. Cell. 2000;101(3):331-40.

25. Fowler S, Lee K, Onouchi H, Samach A, Richardson K, Morris B, et al. GIGANTEA: a circadian clock-controlled gene that regulates photoperiodic flowering in Arabidopsis and encodes a protein with several possible membrane-spanning domains. EMBO J. 1999;18(17):4679-88.

26. Imaizumi T, Schultz TF, Harmon FG, Ho LA, Kay SA. FKF1 F-box protein mediates cyclic degradation of a repressor of CONSTANS in Arabidopsis. Science. 2005:309(5732):293-7.

27. Fornara F, Panigrahi KC, Gissot L, Sauerbrunn N, Rühl M, Jarillo JA, et al. Arabidopsis DOF transcription factors act redundantly to reduce CONSTANS expression and are essential for a photoperiodic flowering response. Dev Cell. 2009:17(1):75-86.

28. Yanovsky MJ, Kay SA. Molecular basis of seasonal time measurement in Arabidopsis. Nature. 2002:419(6904):308-12.

29. Portolés S, Más P. Altered oscillator function affects clock resonance and is responsible for the reduced day-length sensitivity of CKB4 overexpressing plants. Plant J. 2007;51(6):966-77.

30. Sawa M, Kay SA. GIGANTEA directly activates Flowering Locus $T$ in Arabidopsis thaliana. Proc Natl Acad Sci U S A. 2011;108(28):11698-703.

31. Johansson M, Staiger D. SRR1 is essential to repress flowering in noninductive conditions in Arabidopsis thaliana. J Exp Bot. 2014;65(20):5811-22.

32. Kang MY, Yoo SC, Kwon HY, Lee BD, Cho JN, Noh YS, et al. Negative regulatory roles of DE-ETIOLATED1 in flowering time in Arabidopsis. Sci Rep. 2015;5:9728.

33. Park DH, Somers DE, Kim YS, Choy YH, Lim HK, Soh MS, et al. Control of circadian rhythms and photoperiodic flowering by the Arabidopsis GIGANTEA gene. Science. 1999;285(5433):1579-82

34. Staiger D, Allenbach L, Salathia N, Fiechter V, Davis SJ, Millar AJ, et al. The Arabidopsis SRR1 gene mediates phyB signaling and is required for normal circadian clock function. Genes Dev. 2003;17(2):256-68.

35. Alabadí D, Oyama T, Yanovsky MJ, Harmon FG, Más P, Kay SA. Reciprocal regulation between TOC1 and LHY/CCA1 within the Arabidopsis circadian clock. Science. 2011;293(5531):880-3. 
36. Gendron JM, Pruneda-Paz JL, Doherty CJ, Gross AM, Kang SE, Kay SA. Arabidopsis circadian clock protein, TOC1, is a DNA-binding transcription factor. Proc Natl Acad Sci U S A. 2012;109(8):3167-72.

37. Niwa $Y$, Ito S, Nakamichi N, Mizoguchi T, Niinuma K, Yamashino T, et al. Genetic linkages of the circadian clock-associated genes, TOC1, CCA1 and $L H Y$, in the photoperiodic control of flowering time in Arabidopsis thaliana. Plant Cell Physiol. 2007;48(7):925-37.

38. Lu SX, Webb CJ, Knowles SM, Kim SH, Wang Z, Tobin EM. CCA1 and ELF3 interact in the control of hypocotyl length and flowering time in Arabidopsis. Plant Physiol. 2012;158(2):1079-88.

39. Michael TP, Salomé PA, Yu HJ, Spencer TR, Sharp EL, McPeek MA, et al. Enhanced fitness conferred by naturally occurring variation in the circadian clock. Science. 2003:302(5647):1049-53.

40. MacGregor DR, Gould P, Foreman J, Griffiths J, Bird S, Page R, et al. HIGH EXPRESSION OF OSMOTICALLY RESPONSIVE GENES1 is required for circadian periodicity through the promotion of nucleo-cytoplasmic mRNA export in Arabidopsis. Plant Cell. 2013;25(11):4391-404.

41. Carpenter CD, Kreps JA, Simon AE. Genes encoding glycine-rich Arabidopsis thaliana proteins with RNA-binding motifs are influenced by cold treatment and an endogenous circadian rhythm. Plant Physiol. 1994;104(3):1015-25.

42. Millar AJ, Kay SA. Circadian control of cab gene transcription and mRNA accumulation in Arabidopsis. Plant Cell. 1991;3(5):541-50.

43. Scortecci KC, Michaels SD, Amasino RM. Identification of a MADS-box gene, FLOWERING LOCUS M, that represses flowering. Plant J. 2001;26(2):229-36.

44. Fujiwara S, Oda A, Yoshida R, Niinuma K, Miyata K, Tomozoe Y, et al. Circadian clock proteins LHY and CCA1 regulate SVP protein accumulation to control flowering in Arabidopsis. Plant Cell. 2008;20(11):2960-71.

45. Michaels SD, Amasino RM. Loss of FLOWERING LOCUS C activity eliminates the late-flowering phenotype of FRIGIDA and autonomous pathway mutations but not responsiveness to vernalization. Plant Cell. 2001;13(4):935-41.

46. Pruneda-Paz JL, Breton G, Para A, Kay SA. A functional genomics approach reveals CHE as a component of the Arabidopsis circadian clock. Science. 2009;323(5920):1481-5.

47. Schaffer R, Ramsay N, Samach A, Corden S, Putterill J, Carré IA, et al. The late elongated hypocotyl mutation of Arabidopsis disrupts circadian rhythms and the photoperiodic control of flowering. Cell. 1998:93(7):1219-29.

48. Lu SX, Knowles SM, Andronis C, Ong MS, Tobin EM. CIRCADIAN CLOCK ASSOCIATED1 and LATE ELONGATED HYPOCOTYL function synergistically in the circadian clock of Arabidopsis. Plant Physiol. 2009;150(2):834-43.

49. Seaton DD, Smith RW, Song YH, MacGregor DR, Stewart K, Steel G, et al. Linked circadian outputs control elongation growth and flowering in response to photoperiod and temperature. Mol Syst Biol. 2015;11(1):776.

50. Knowles SM, Lu SX, Tobin EM. Testing time: can ethanol-induced pulses of proposed oscillator components phase shift rhythms in Arabidopsis? J Biol Rhythms. 2008;23(6):463-71.

51. Green RM, Tobin EM. Loss of the circadian clock-associated protein 1 in Arabidopsis results in altered clock-regulated gene expression. Proc Natl Acad Sci U S A. 1999;96(7):4176-9.

52. Wang W, Barnaby JY, Tada Y, Li H, Tör M, Caldelari D, et al. Timing of plant immune responses by a central circadian regulator. Nature. 2011; 470(7332):110-4.

53. Burgess A, Searle I. The clock primes defense at dawn. Immunol Cell Biol. 2011;89(6):661-2.

54. Dai S, Wei X, Pei L, Thompson RL, Liu Y, Heard JE, et al. BROTHER OF LUX ARRHYTHMO is a component of the Arabidopsis circadian clock. Plant Cell. 2011;23(3):961-72.

55. Yakir E, Hilman D, Kron I, Hassidim M, Melamed-Book N, Green RM. Posttranslational regulation of CIRCADIAN CLOCK ASSOCIATED1 in the circadian oscillator of Arabidopsis. Plant Physiol. 2009;150(2):844-57.

56. Takada S, Goto K. TERMINAL FLOWER2, an Arabidopsis homolog of heterochromatin protein1, counteracts the activation of FLOWERING LOCUS $T$ by CONSTANS in the vascular tissues of leaves to regulate flowering time. Plant Cell. 2003;15(12):2856-65.

57. Kwon YJ, Park MJ, Kim SG, Baldwin IT, Park CM. Alternative splicing and nonsense-mediated decay of circadian clock genes under environmental stress conditions in Arabidopsis. BMC Plant Biol. 2014;14:136.

58. Udvardi MK, Czechowski T, Scheible WR. Eleven golden rules of quantitative RT-PCR. Plant Cell. 2008;20(7):1736-7.

59. Jung JH, Lee S, Yun J, Lee M, Park CM. The miR172 target TOE3 represses AGAMOUS expression during Arabidopsis floral patterning. Plant Sci. 2014; 215-216:29-38.
60. Yoo SD, Cho YH, Sheen J. Arabidopsis mesophyll protoplasts: a versatile cell system for transient gene expression analysis. Nat Protoc. 2007;2(7):1565-72.

61. Jefferson RA, Kavanagh TA, Bevan MW. GUS fusions: beta-glucuronidase as a sensitive and versatile gene fusion marker in higher plants. EMBO J. 1987; 6(13):3901-7.

62. Park J, Kim YS, Kim SG, Jung JH, Woo JC, Park CM. Integration of auxin and salt signals by the NAC transcription factor NTM2 during seed germination in Arabidopsis. Plant Physiol. 2011;156(2):537-49.

63. Seo PJ, Park MJ, Lim MH, Kim SG, Lee M, Baldwin IT, et al. A self-regulatory circuit of CIRCADIAN CLOCK-ASSOCIATED1 underlies the circadian clock regulation of temperature responses in Arabidopsis. Plant Cell. 2012;24(6): 2427-42.

\section{Submit your next manuscript to BioMed Central and we will help you at every step:}

- We accept pre-submission inquiries

- Our selector tool helps you to find the most relevant journal

- We provide round the clock customer support

- Convenient online submission

- Thorough peer review

- Inclusion in PubMed and all major indexing services

- Maximum visibility for your research

Submit your manuscript at www.biomedcentral.com/submit
Biomed Central 